\title{
The Effects of Rumination and Depressive Symptoms on the Prediction of Negative Attributional Style Among College Students
}

\author{
Cola S. L. Lo · Samuel M. Y. Ho · Steven D. Hollon
}

Published online: 3 February 2009

(C) Springer Science+Business Media, LLC 2009

\begin{abstract}
Research on cognitive vulnerability to depression has identified negative cognitive style and rumination as distinct risk factors for depression but how rumination would influence negative cognitive style remains unclear. The present study investigated the relationship between rumination and negative attributional style and specifically tested the potential moderating effect of depressive symptoms and processing mode during rumination on activating negative attributional style. After completing the baseline measures of depressive symptoms, dysphoric affect, and negative attributional style, participants were randomly assigned to three experimental conditions: analytical selffocus, experiential self-focus, and distraction, in which the degree of self-focus and mode of processing were manipulated. A second set of mood and cognitive measures was administered afterwards. Results showed that a stronger positive relationship between negative attributional style and level of depressive symptoms was found in the analytical self-focus condition, relative to the experiential and distraction conditions. This finding suggested that processing mode in rumination interacted with depressive symptoms to predict negative attributional style.
\end{abstract}

Keywords Rumination - Depression .

Negative attributional style

C. S. L. Lo $(\bowtie) \cdot$ S. M. Y. Ho

Department of Psychology, The University of Hong Kong,

Pokfulam, Hong Kong

e-mail: colalo@cuhk.edu.hk

S. D. Hollon

Department of Psychology, Vanderbilt University,

Nashville, TN, USA

\section{Introduction}

Cognitive theories of depression state that people have characteristic ways of understanding negative life events and that those who exhibit a dispositional negative cognitive style and dysfunctional attitudes are at greater risk for depression (Abramson et al. 1989; Beck 1987). The hopelessness theory of depression (Abramson et al. 1989) postulates that depressive symptoms are likely to occur when negative life events are attributed to stable and global causes, when they are perceived as being associated with other negative consequences in the future, and construed as implying personal deficit and worthlessness. Considerable empirical support shows that the negative cognitive style featured in the hopelessness theory, especially in interaction with stressors, predicts prospective depressive symptoms and clinically significant depressive disorders (Abramson et al. 2002; Hankin et al. 2004, 2005; Scher et al. 2005).

Rumination is another cognitive risk factor for depression that has received growing attention in the literature. According to the response style theory (Nolen-Hoeksema 1991), rumination is defined as a mode of thinking that involves repetitively and passively focusing on one's symptoms of depression as well as on the causes and consequences of those symptoms. The theory proposes that individuals have dispositional differences in the way they react to negative mood states and those who respond to a depressed mood by consistently engaging in rumination tend to have more persistent and severe depressive episodes. In contrast, responses that serve to distract one from depressed mood are posited to alleviate feelings of sadness. Although the original theory suggested that rumination should predict the duration of depressed mood or depressive episodes, recent evidence suggests that rumination also predicts new onsets of major depressive episodes (Just 
and Alloy 1997; Nolen-Hoeksema 2000; Nolen-Hoeksema et al. 1994). Experimental studies have shown that rumination (relative to distraction) intensified negative mood states, enhanced negative thinking and memory, and impaired social problem solving in dysphoric individuals (see Lyubomirsky and Tkach 2004 for a review). However, similar effects did not observe among nondysphoric individuals, suggesting that it is the combination of dysphoria and rumination that contributes to the negative outcomes in rumination (Lyubomirsky and Nolen-Hoeksema 1995).

A recent study found that negative cognitive style and rumination represent empirically distinct (albeit highly correlated) cognitive risk factors for depression (Hankin et al. 2007). In an attempt to conceptualize the relationship between negative cognitive style and rumination in depression, Abramson et al. (2002) proposed that cognitively vulnerable individuals should be at higher risk for engaging in rumination as their underlying negative thinking makes it very difficult to exit the self-regulatory cycle. Empirical data support that individuals who exhibit a negative cognitive style and have the tendency to ruminate would be more likely than others to have more severe depressive episodes (Alloy et al. 2000; Robinson and Alloy 2003). Besides, rumination has been found to mediate the relationship between depression and negative cognitive style, as well as dysfunctional attitudes and neuroticism, suggesting that rumination may represent a common mechanism through which a variety of risk factors affect depression (Lo et al. 2008; Roberts et al. 1998; Spasojevic and Alloy 2001). Along with the negative cognitive consequences of rumination that have been found in the experimental studies, rumination and negative cognitive style may be dynamically related and their relationship may be reciprocal in nature. The presence of rumination would increase the influence of negative cognition on depression and this, in turn, would increase the influence of depression on cognition in a way that a self-perpetuated cycle of cognitive-affective processing would be generated during depression (Ciesla and Roberts 2007; Teasdale 1999).

Recent evidence has suggested that the consequences of rumination could be moderated by the mode of thinking adopted in times of distress. Two distinct modes of selffocus during rumination have been identified that have distinct functional properties with respect to depression (Watkins and Moulds 2005; Watkins and Teasdale 2004). The abstract analytical processing mode is focused on evaluating higher level causes, meanings, consequences, and implications of self-experience. In contrast, the concrete experiential processing mode is focused on the lower level, specific, and direct experience of one's thoughts, feelings, and sensations in the present moment. The theoretical rationale for this distinction comes from the reduced concreteness theory (Borkovec et al. 1998; Stober and Borkovec
2002) and the interacting cognitive subsystems theory (Teasdale 1999). Both these theories propose that abstract analytical processing at times of negative self-experience is maladaptive in depression since it is associated with poorer emotional processing and overgeneralization (Ganellen 1988; Teasdale 1999). The abstract analytical processing may also provide event descriptions that are less detailed and conceptual that might hinder effective problem solving.

Research findings showed that among depressed patients, an induction of analytical self-focus (the abstract analytical mode) reduced the specificity of autobiographical memory recall (Watkins and Teasdale 2001, 2004), impaired social problem solving (Watkins and Moulds 2005), and increased endorsement of global negative self-judgments (Rimes and Watkins 2005) compared to experiential self-focus (the concrete experiential mode). Consistently, such differential effect was not evident among nondepressed participants, suggesting that the presence of depressive symptoms would be necessary to trigger the negative effect of rumination. These findings provide support to the mode of processing hypothesis (Watkins and Moulds 2005) that it is the processing mode, and not the degree of self-focus, that influences cognitive consequences in depression. However, since these studies did not include a distraction condition, it would be difficult to draw conclusions regarding the different predictions of processing mode hypothesis and the degree of self-focus hypothesis. Given that abstract analytical processing would facilitate overgeneralization, it is speculated that reliance on an abstract analytical processing mode during rumination would also amplify and intensify the underlying negative cognitive style (as featured in the hopelessness theory of depression) in individuals who are experiencing depressive symptoms.

In summary, research evidence suggests the presence of depressive symptoms and the analytical mode of processing during rumination would activate negative cognitive style. However, little research has directly investigated how these variables may act together to enhance the effect of negative cognitive style. It is important to examine the potential moderating effect directly so as to shed light on the dynamic relationship among these factors. It is also imperative to examine how these vulnerability factors interrelate in order to more fully understand the mechanisms leading to depression, and thus identify the most appropriate points for intervention and guide the development of even more efficacious treatments of depression.

The present experimental study investigated the moderating effect of depressive symptoms and the processing mode in rumination on activating negative attributional style (the negative inferences about the causes of negative events). In accordance with the processing mode hypothesis (Watkins and Moulds 2005), mode of processing during rumination, and not the degree of self-focus would be 
associated with the level of negative attributional style. In addition to manipulating the mode of processing, a distraction condition was included as a reference condition so that the differential effects of processing mode and degree of self-focus could be directly examined. It was hypothesized that the level of depressive symptoms would interact with the mode of processing in predicting negative attributional style. Specifically, it was predicted that a stronger association between depressive symptoms and negative attributional style would be found in the analytical self-focus condition (a maladaptive mode of processing) than would be found in the experiential self-focus and distraction conditions.

\section{Method}

\section{Participants}

The participants were undergraduate students at The University of Hong Kong who participated in the study in return for research credit. The sample comprised 23 male and 49 female participants with a mean age of 19.47 $(\mathrm{SD}=1.37)$. The study received ethical approval from the Ethics Committee of the Psychology Department at The University of Hong Kong.

\section{Materials}

\section{Beck Depression Inventory-II (BDI-II; Beck et al. 1996)}

The BDI-II is a 21-item self-report measure of depressive symptoms that possesses strong psychometric properties. The Chinese version of the BDI-II (C-BDI-II; Chinese Behavioral Sciences Society 2000) was used in this study. It has been reported to have strong psychometric properties and an internal consistency of .94 in a Chinese sample (Byrne et al. 2004). In the present sample, the coefficient alpha was .82 .

\section{Visual Analogue Scale (VAS; Teasdale and Dent 1987)}

The VAS was used to measure current level of dysphoric mood. Participants rated current "in the moment" feelings of sad/depressed emotions on scales ranging from 0 (not at all sad/depressed) to 100 (extremely sad/depressed) with anchors at every 10 points along the scale.

\section{Attributional Style Questionnaire (ASQ; Peterson et al.} 1982)

The ASQ is a self-report measure that assesses causal attributions for six hypothetical positive and negative events along the dimensions of internality, stability, and globality on a 7-point Likert scale. A composite negative score can be computed by averaging the values of the respondent's responses on the six negative events to produce a score that ranges from 1 to 7 . A parallel version of the ASQ comprising another six hypothetical negative events, matched in length and content to the ASQ, was adapted from the expanded attributional style questionnaire (EASQ; Peterson and Villanova 1988). These two parallel versions of the ASQ were used as repeated measures of negative attributional style and were counterbalanced within each condition for time of measurement. These measures of negative attributional style were translated into Chinese using the translation and back translation procedure. A pilot study with 110 college students revealed that these two versions of the ASQ had comparable means and standard deviations, and were highly correlated $(r=.73$, $p<.001)$. Internal consistencies for the two measures were satisfactory ( $\alpha=.81$ and .80 ). The hopelessness theory of depression (Abramson et al. 1989) has de-emphasized the importance of the internality dimension, and demonstrated that generality, a composite score computed from the stable and global items, may show a stronger relationship to depression than does the traditional internal, stable, and global composite. Past research reported satisfactory internal consistency with the generality score, the alphas of which ranged from .67 to .77 (Fresco et al. 2006; Metalsky et al. 1987). The generality score was used to index negative attributional style in this study. In the present sample, coefficient alphas for the generality score (ASQ-GEN) were .75 at Time 1 and .81 at Time 2 .

\section{Experimental Conditions}

The three manipulated conditions were designed to influence the degree of self-focus and processing mode of thinking by requiring the participants to focus their attention on a series of 45 items presented in written form in Chinese for $8 \mathrm{~min}$ (adapted from Nolen-Hoeksema and Morrow 1993). For the two rumination conditions, 45 identical items that were symptom-focused, emotionfocused and self-focused were used but with different sets of instruction for manipulating the modes of processing (adapted from Watkins and Teasdale 2004). Instructions for the analytical self-focus condition emphasized thinking about the causes, meanings, and consequences of each item whereas the instructions for the experiential self-focus condition emphasized focusing one's attention on the experience of each item. The distraction condition required participants to focus their attention externally on thoughts that were not related to self. Nolen-Hoeksema and Morrow (1993) reported that items in both the rumination and the distraction conditions were rated as being equally neutral in affective tone by nondysphoric judges. The translation and 
back translation procedure was used to develop the Chinese instructions and items for the manipulated conditions.

\section{Procedure}

After the participants had given their written informed consent, they completed the BDI-II and baseline measures of the VAS and the ASQ-GEN. The experimenter then introduced and explained the manipulation task. The participants were randomly assigned to one of the three manipulated conditions (analytical self-focus, experiential self-focus, and distraction), and were told to spend exactly $8 \mathrm{~min}$ on the assigned task. Following the manipulation, the participants completed the second set of the VAS and the ASQ-GEN. Finally, the participants filled out a questionnaire asking open-ended questions regarding the purpose of the study. They were then thoroughly debriefed. The entire procedure lasted approximately $45 \mathrm{~min}$.

\section{Results}

\section{Preliminary Analyses}

Random assignment of the participants to the three conditions resulted in 24 participants in the analytical selffocus condition, 25 in the experiential self-focus condition, and 23 in the distraction condition. A one-way ANOVA revealed that the three groups were equivalent in their level of depressive symptoms, dysphoric affect, and negative attributional style prior to the manipulation. Descriptive statistics and correlations of the variables are presented in Table 1 . The BDI-II correlated significantly with VAS and ASQ-GEN at the baseline, suggesting that participants with

Table 1 Means, standard deviations, and correlations among variables

\begin{tabular}{lccccc}
\hline Variables & 1 & 2 & 3 & 4 & 5 \\
\hline 1. BDI-II & - & & & & \\
2. VAS-Time 1 & $.66^{* * *}$ & - & & & \\
3. VAS-Time 2 & $.60 * * *$ & $.82 * * *$ & - & & \\
4. ASQ-GEN-Time 1 & $.40^{* * *}$ & $.37 * *$ & $.46 * * *$ & \\
5. ASQ-GEN-Time 2 & $.31 * *$ & .18 & $.26 *$ & $.68 * * *$ & - \\
Mean & 10.74 & 33.26 & 32.78 & 4.34 & 4.41 \\
SD & 6.12 & 26.17 & 25.71 & 0.73 & 0.87 \\
\hline
\end{tabular}

Note BDI-II Beck depression inventory-second edition, VAS selfreport of sad/depressed mood on a $0-100$ visual analogue scale, $A S Q$ GEN composite score of generality averaging the respondent's responses on six negative hypothetical events along the dimensions of stability and globality

$* P<.05, * * P<.01, * * * P<.001$ more depressive symptoms also reported more dysphoric affect and negative attributional style prior to the manipulation. Since there was no significant effect of gender and age on the dependent variables, all reported analyses were conducted by collapsing across these variables.

\section{Regression Analyses}

Multiple linear regressions with forced entry were used to test the main effects of the manipulated conditions and the BDI-II, as well as their interactions, in predicting the dysphoric affect and negative attributional style at postmanipulation. There were two independent variables in the equations: (1) level of depressive symptoms (BDI-II) was centered and treated as a continuous variable (Aiken and West 1991); (2) the manipulated conditions were treated as a categorical variable with three levels: analytical self-focus, experiential self-focus, and distraction. The three level categorical variable was then converted into two contrast-coded variables. FOCUS compared the effects of the two self-focus conditions with that of distraction, and MODE compared the effects of analytical self-focus with that of experiential self-focus. To evaluate the interaction effect of depressive symptoms, two interaction terms were created by multiplying the BDI-II scores by the contrastcoded variables, FOCUS and MODE. Time 2 scores of the VAS and the ASQ-GEN were the dependent measures in each equation. The respective Time 1 measure was entered as a covariate in the first step of the regression equation, followed by the contrast-coded variables along with the BDI-II. The final step added the corresponding interaction terms. Owing to the relatively small sample size within each condition, influence statistics were performed to check for outliers. Cook's $D$ was satisfactory (with values of $<0.3$ for all cases) in the regression equations, indicating that no outlier had an undue influence on the results. An alpha level of 0.05 was used for all statistical tests (2-tailed).

\section{Effects of the BDI-II and Manipulations on the VAS}

Table 2 presents the results of the moderated regression analysis on the VAS (Time 2). Postmanipulation levels of dysphoric affect were associated with FOCUS $(\beta=.52$, $p<.001$ ), but were not associated with either MODE or BDI-II. The interaction terms of FOCUS $\times$ BDI-II and MODE $\times$ BDI-II were also not significant after controlling for Time 1 VAS. These results suggested that relative to the distraction condition, individuals allocated to the two self-focus conditions were more dysphoric after the manipulation regardless of levels of depression. As predicted, the two modes of processing were not associated with the VAS at Time 2 since they were hypothesized to have equivalent effects on negative mood states. 
Table 2 Summary of hierarchical regression analyses for Time 2 measures on the VAS and ASQ-GEN

\begin{tabular}{|c|c|c|c|c|c|}
\hline Predictors & B & SE & $B$ & $t$ & $\Delta R^{2}$ \\
\hline \multicolumn{6}{|l|}{ VAS at Time 2} \\
\hline Step 1 & & & & & $.67 * * *$ \\
\hline Time 1 VAS & 0.81 & 0.07 & 0.82 & $12.01 * * *$ & \\
\hline Step 2 & & & & & $.09 * * *$ \\
\hline Time 1 VAS & 0.80 & 0.08 & 0.81 & $10.16 * * *$. & \\
\hline BDI-II & 0.29 & 0.33 & 0.07 & 0.87 & \\
\hline FOCUS & 15.85 & 3.29 & 0.29 & $4.81 * * *$ & \\
\hline MODE & -2.68 & 3.70 & -0.04 & -0.72 & \\
\hline Step 3 & & & & & .00 \\
\hline Time 1 VAS & 0.80 & 0.08 & 0.82 & $9.78 * * *$ & \\
\hline BDI-II & 0.22 & 0.36 & 0.05 & 0.61 & \\
\hline FOCUS & 15.96 & 3.34 & 0.29 & $4.78 * * *$ & \\
\hline MODE & -2.74 & 3.75 & -0.04 & -0.73 & \\
\hline FOCUS × BDI-II & 0.31 & 0.63 & 0.03 & 0.49 & \\
\hline MODE $\times$ BDI-II & -0.22 & 0.61 & -0.02 & -0.36 & \\
\hline \multicolumn{6}{|l|}{ ASQ-GEN at Time 2} \\
\hline Step 1 & & & & & $.46^{* * *}$ \\
\hline Time 1 ASQ-GEN & 0.82 & 0.11 & 0.68 & $7.79 * * *$ & \\
\hline Step 2 & & & & & .02 \\
\hline Time 1 ASQ-GEN & 0.77 & 0.12 & 0.64 & $6.45^{* * *}$ & \\
\hline BDI-II & 0.01 & 0.01 & 0.05 & 0.52 & \\
\hline FOCUS & 0.21 & 0.17 & 0.12 & 1.28 & \\
\hline MODE & 0.03 & 0.19 & 0.02 & 0.16 & \\
\hline Step 3 & & & & & $.06^{*}$ \\
\hline Time 1 ASQ-GEN & 0.81 & 0.12 & 0.67 & $7.00 * * *$ & \\
\hline BDI-II & 0.01 & 0.01 & 0.04 & 0.45 & \\
\hline FOCUS & 0.18 & 0.16 & 0.10 & 1.12 & \\
\hline MODE & 0.01 & 0.18 & 0.01 & 0.06 & \\
\hline FOCUS $\times$ BDI-II & 0.06 & 0.03 & 0.18 & $1.99^{\#}$ & \\
\hline MODE $\times$ BDI-II & 0.07 & 0.03 & 0.21 & $2.31 *$ & \\
\hline
\end{tabular}

Note BDI-II centered scores according to the recommendation of Aiken and West (1991), FOCUS contrast-coded variable comparing the two self-focus conditions with the distraction condition, $M O D E$ contrast-coded variable comparing analytical self-focus with experiential self-focus

$* P<.05, * * * P<.001,{ }^{\#} P=.051$

Effects of the BDI-II and Manipulations on the ASQ-GEN

Another moderated regression analysis on the ASQ-GEN (Time 2) revealed a significant MODE $\times$ BDI-II interaction term $(\beta=.21, p=.02)$ and the FOCUS $\times$ BDI-II interaction also showed a marginal trend $(\beta=.18$, $p=.051)$ after controlling for the baseline ASQ-GEN (Table 2). There was no main effect of FOCUS or MODE. Simple slope analyses showed that higher ASQ-GEN (Time 2) scores were associated with higher BDI-II scores

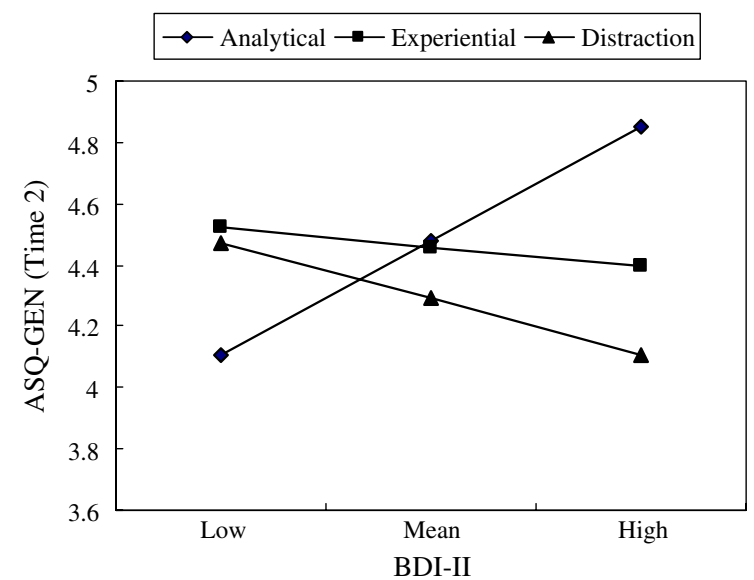

Fig. 1 Simple regression lines depicting the relationship between ASQ-GEN (Time 2) and BDI-II for the three experimental groups conditioned at the mean value of the baseline ASQ-GEN. The value of BDI-II is plotted at one standard deviation below the mean (Low), the mean (Mean) and one standard deviation above the mean (High)

when conditioned at the analytical self-focus condition $[t$ (2.25), $p=.03$ ], but was not associated with BDI-II scores when conditioned at the experiential self-focus and distraction condition. This interaction is plotted in Fig. 1. Subsequent exploratory analyses using dummy-coded variables confirmed that a stronger positive relationship between BDI-II and ASQ-GEN was found when conditioned at the analytical condition relative to the experiential self-focus condition $(\beta=-.33, p=.02)$ and the distraction condition $(\beta=-.30, \quad p=.01)$. These results suggested that the mode of processing interacted with depressive symptoms to predict negative attributional style. These analyses were repeated with mood change as a control. The same patterns of results were obtained, suggesting that these interaction effects were independent of the affective change during the manipulation.

\section{Discussion}

This study examined the relationship between rumination and negative attributional style with the hypothesis that the effect of rumination on negative attributional style would depend on both the level of depressive symptoms and mode of processing during rumination. The present findings provide support for this prediction and suggest that the processing mode in rumination interacts with depressive symptoms to predict negative attributional style.

Specifically, the results revealed a stronger association between depressive symptoms and negative attributional style in the analytical self-focus condition, relative to the experiential self-focus and the distraction conditions. These findings indicate that ruminating in an abstract analytical 
mode activates and intensifies the underlying negative attributional style in people who are experiencing higher levels of depressive symptoms. Given that a weaker association between depressive symptoms and negative attributional style was found in the experiential self-focus and distraction conditions, the processing mode hypothesis was directly supported that it is the mode of processing during self-focus, and not the degree of self-focus that determines the negative cognitive outcomes in depression (Watkins and Moulds 2005).

Previous studies suggest that both rumination and negative cognitive style predict depressive symptoms (Alloy et al. 2000; Ciesla and Roberts 2007; Robinson and Alloy 2003) and similar patterns of findings were also obtained in this sample. ${ }^{1}$ The results of the present study, along with the previous research, demonstrate that the presence of depressive symptoms (which may have been initially caused by a negative cognitive style), may further increase the accessibility of negative attributional style through the negative effects of ruminative processing (Ciesla and Roberts 2007; Teasdale 1999). Past research suggests that cognitively vulnerable individuals are at higher risk for engaging in rumination and the present results extend the finding that ruminating in an abstract analytical mode further facilitates the access to negative attributional style. It is important to note that such findings did not suggest rumination creates or causes the negative attributional style, but did suggest that it makes the effect more available to those with elevated levels of depressive symptoms. Given that the negative effect of abstract analytical processing has been found in social problem solving, overgeneral memory, and global negative self-judgments, and that it further extends to the negative attributional style that characterizes depressed people, rumination in the mode of abstract analytical processing might be a critical proximal mechanism that contributes to depression by creating a vicious cycle of cognitive-affective processing in times of dysphoria (Teasdale 1999). The findings also provide further evidence for the proposed reciprocal and dynamic relationship among rumination, negative cognitive style, and depressive symptoms. Nevertheless, owing to the preliminary nature of the findings, future longitudinal research is needed to further elucidate the direction and nature of the causal relationships among these variables.

\footnotetext{
${ }^{1}$ Although this was not the primary hypothesis of the study, for exploratory purpose, a regression analysis testing the effects of rumination and negative attributional style on changes in dysphoric mood was conducted with results indicating main effects of FOCUS $(\beta=.90, p<.05)$ and ASQ-GEN $(\beta=.15, p<.05)$ with a nonsignificant interaction effect $(p=.12)$, suggesting the importance of these variables in worsening depressive mood states.
}

Although negative cognitive style is considered to be a static, trait-like feature in depression-prone individuals, the present findings showed that the effect of negative attributional style on depression might depend upon the dynamic activation of an abstract-analytical mode of selffocus in times of distress (Watkins and Teasdale 2004). Furthermore, previous research has demonstrated that the effect of negative attributional style (generality score of the ASQ) could be responsive to a mood priming challenge among dysphoric students with a history of depression (Fresco et al. 2006). Although the vulnerability-stress model posits that the effect of negative cognitive style on depression can be triggered by negative life events, these findings suggest that the underlying cognitive vulnerability could also be activated by internal affective and cognitive processes such as negative mood states and ruminative processing (Scher et al. 2005).

From the clinical perspective, the current findings suggest that both negative attributional style and the abstract analytical mode of ruminative processing are important cognitive factors related to depression. Although standard cognitive behavioral therapy for depression primarily targets changing dysfunctional thought contents, which is necessary and important, one potential way to improve the treatment efficacy might be to modify maladaptive ruminative processing as well. It may be important to help depressed people disengage from the analytical mode of processing that is habitually activated in times of distress. Although distraction could be effective to alleviate transient sad mood states, the effect of experiential self-focus implies that teaching depressed people to adopt a nonanalytical form of self-focus might both facilitate emotional processing and improve problem solving in a manner that would contribute to the treatment process (Ma and Teasdale 2004).

Finally, although we have demonstrated the differential effect between rumination and distraction on dysphoric affect, we were unable to replicate the findings from adult samples that these effects on mood are dependent on the pre-existing level of depressive symptoms. It may be possible that younger participants may be more labile and changeable in mood and that their affective responses to rumination manipulation would be different. Similar findings have been reported in an adolescent sample that both the depressed and control groups of participants showed similar increase in negative mood after a rumination induction (Park et al. 2004).

There are some limitations that need to be addressed. First, the sample size was relatively small, and the fact that the participants exhibited only a mild to a moderate level of depression limits the extent to which the findings can be generalized to a clinical population. Second, we did not have a manipulation check associated with the 
experimental tasks and thus we can only infer the behavior of participants during the manipulation process. Last, future studies should consider using more sophisticated methodologies when assessing the effects of rumination in laboratory to diminish the potential influence of demand and response bias effects.

Acknowledgments We gratefully acknowledge the help of Professor Susan Nolen-Hoeksema, Dr. Ed Watkins, and Dr. Katharine Rimes for providing experimental materials and protocols for this study.

\section{References}

Abramson, L. Y., Alloy, L. B., Hankin, B. L., Haeffel, G. J., MacCoon, D. G., \& Gibb, B. E. (2002). Cognitive vulnerabilitystress models of depression in a self-regulatory and psychobiological context. In I. H. Gotlib \& C. L. Hammen (Eds.), Handbook of depression. New York: Guilford.

Abramson, L. Y., Metalsky, G. I., \& Alloy, L. B. (1989). Hopelessness depression: A theory-based subtype of depression. Psychological Review, 96, 358-372. doi:10.1037/0033-295X.96. 2.358 .

Aiken, L. S., \& West, S. G. (1991). Multiple regression: Testing and interpreting interactions. California: Sage.

Alloy, L. B., Abramson, L. Y., Hogan, M. E., Whitehouse, W. G., Rose, D. T., Robinson, M. S., et al. (2000). The Temple-Wisconsin cognitive vulnerability to depression (CVD) project: Lifetime history of axis I psychopathology in individuals at high and low cognitive vulnerability to depression. Journal of Abnormal Psychology, 109, 403-418. doi:10.1037/0021-843X.109.3.403.

Beck, A. T. (1987). Cognitive models of depression. Journal of Cognitive Psychotherapy, 1, 5-37.

Beck, A. T., Steer, R. A., \& Brown, G. K. (1996). Beck depression inventory manual (2nd ed.). Texas: Psychological Corporation.

Borkovec, T. D., Ray, W. J., \& Stober, J. (1998). Worry: A cognitive phenomenon intimately linked to affective, physiological and interpersonal behavioral processes. Cognitive Therapy and Research, 8, 561-576. doi:10.1023/A:1018790003416.

Byrne, B., Stewart, S. M., \& Lee, P. W. H. (2004). Validating the beck depression inventory-II for Hong Kong community adolescents. International Journal of Testing, 4, 199-216. doi: 10.1207/s15327574ijt0403_1.

Chinese Behavioral Sciences Society. (2000). The Chinese version of the beck depression inventory (2nd ed.). New York: Harcourt Brace.

Ciesla, J. A., \& Roberts, J. E. (2007). Rumination, negative cognition, and their interactive effects on depressed mood. Emotion (Washington, DC), 7, 555-565. doi:10.1037/1528-3542.7.3.555.

Fresco, D. M., Heimberg, R. G., Abramowitz, A., \& Bertram, T. L. (2006). The effect of a negative mood priming challenge on dysfunctional attitudes, explanatory style, and explanatory flexibility. The British Journal of Clinical Psychology, 45, 167-183. doi:10.1348/014466505X35137.

Ganellen, R. J. (1988). Specificity of attributions and overgeneralization in depression and anxiety. Journal of Abnormal Psychology, 97, 83-86. doi:10.1037/0021-843X.97.1.83.

Hankin, B. L., Abramson, L. Y., Miller, N., \& Haeffel, G. J. (2004). Cognitive vulnerability-stress theories of depression: Examining affective specificity in the prediction of depression versus anxiety in 3 prospective studies. Cognitive Therapy and Research, 28, 309-345. doi:10.1023/B:COTR.0000031805.60529.0d.
Hankin, B. L., Fraley, R. C., \& Abela, J. R. Z. (2005). Daily depressed and cognitions about stress: Evidence for a trait-like depressogenic cognitive style and the prediction of depressive symptoms trajectories in a prospective daily diary study. Journal of Personality and Social Psychology, 88, 673-685. doi:10.1037/ 0022-3514.88.4.673.

Hankin, B. L., Lakdawalla, Z., Carter, I. L., Abela, J. R. Z., \& Adams, P. (2007). Are neuroticism, cognitive vulnerabilities and selfesteem overlapping or distinct risks for depression? Evidence from exploratory and confirmatory factor analyses. Journal of Social and Clinical Psychology, 26, 29-63. doi:10.1521/jscp. 2007.26.1.29.

Just, N., \& Alloy, L. B. (1997). The response styles theory of depression: Tests and extension of the theory. Journal of Abnormal Psychology, 106, 221-229. doi:10.1037/0021-843X. 106.2.221

Lo, C. S. L., Ho, S. M. Y., \& Hollon, S. D. (2008). The effects of rumination and negative cognitive styles on depression: A mediation analysis. Behaviour Research and Therapy, 46, 487495. doi:10.1016/j.brat.2008.01.013.

Lyubomirsky, S., \& Nolen-Hoeksema, S. (1995). Effect of selffocused rumination on negative thinking and interpersonal problem solving. Journal of Personality and Social Psychology, 69, 176-190. doi:10.1037/0022-3514.69.1.176.

Lyubomirsky, S., \& Tkach, C. (2004). The consequences of dysphoric rumination. In C. Papageorgiou \& A. Wells (Eds.), Depressive rumination: Nature, theory and treatments (pp. 21-42). London, UK: Wiley.

Ma, H., \& Teasdale, J. D. (2004). Mindfulness-based cognitive therapy for depression: Replication and exploration of differential relapse prevention effects. Journal of Consulting and Clinical Psychology, 72, 31-40. doi:10.1037/0022-006X.72.1.31.

Metalsky, G. I., Halberstadt, L. J., \& Abramson, L. Y. (1987). Vulnerability to depressive mood reactions: Toward a more powerful test of the diathesis-stress and causal mediation components of the reformulated learned helplessness theory. Journal of Personality and Social Psychology, 52, 386-393. doi: 10.1037/0022-3514.52.2.386.

Nolen-Hoeksema, S. (1991). Responses to depression and their effects on the duration of depressive episodes. Journal of Abnormal Psychology, 100, 569-582. doi:10.1037/0021-843X.100.4.569.

Nolen-Hoeksema, S. (2000). The role of rumination in depressive disorders and mixed anxiety/depressive symptoms. Journal of Abnormal Psychology, 109, 504-511. doi:10.1037/0021-843X. 109.3.504.

Nolen-Hoeksema, S., \& Morrow, J. (1993). Effects of rumination and distraction on naturally occurring depressive mood. Cognition and Emotion, 7, 561-570. doi:10.1080/02699939308409206.

Nolen-Hoeksema, S., Parker, L. E., \& Larson, J. (1994). Ruminative coping with depressed mood following loss. Journal of Personality and Social Psychology, 67, 92-104. doi:10.1037/00223514.67.1.92.

Park, R. J., Goodyear, I. M., \& Teasdale, J. D. (2004). Effects of induced rumination and distraction on mood and overgeneral autobiographical memory in adolescent major depressive disorder and controls. Journal of Child Psychology and Psychiatry and Allied Disciplines, 45, 996-1006. doi:10.1111/j.1469-7610. 2004.t01-1-00291.x.

Peterson, C., Semmel, A., von Baeyer, C., Abramson, L. Y., Metalsky, G. I., \& Seligman, M. E. P. (1982). The attributional style questionnaire. Cognitive Therapy and Research, 6, 287299. doi:10.1007/BF01173577.

Peterson, C., \& Villanova, P. (1988). An expanded attributional style questionnaire. Journal of Abnormal Psychology, 97, 87-89. doi: 10.1037/0021-843X.97.1.87. 
Rimes, K. A., \& Watkins, E. (2005). The effects of self-focused rumination on global negative self-judgments in depression. Behaviour Research and Therapy, 43, 1673-1681. doi:10.1016/ j.brat.2004.12.002.

Roberts, J. E., Gilboa, E., \& Gotlib, I. H. (1998). Ruminative response style and vulnerability to episodes of dysphoria: Gender, neuroticism, and episode duration. Cognitive Therapy and Research, 22, 401-423. doi:10.1023/A:1018713313894.

Robinson, M. S., \& Alloy, L. B. (2003). Negative cognitive styles and stress-reactive rumination interact to predict depression: A prospective study. Cognitive Therapy and Research, 27, 275291. doi:10.1023/A:1023914416469.

Scher, C. D., Ingram, R. E., \& Segal, Z. V. (2005). Cognitive reactivity and vulnerability: Empirical evaluation of construct activation and cognitive diatheses in unipolar depression. Clinical Psychology Review, 25, 487-510. doi:10.1016/j.cpr. 2005.01.005.

Spasojevic, J., \& Alloy, L. B. (2001). Rumination as a common mechanism relating depressive risk factors to depression. Emotion (Washington, DC), 1, 25-37. doi:10.1037/1528-3542. 1.1.25.
Stober, G., \& Borkovec, T. D. (2002). Reduced concreteness of worry in generalized anxiety disorder: Findings from a therapy study. Cognitive Therapy and Research, 26, 89-96. doi:10.1023/ A: 1013845821848.

Teasdale, J. D. (1999). Emotional processing, three modes of mind and the prevention of relapse in depression. Behaviour Research and Therapy, 37, 553-577.

Teasdale, J. D., \& Dent, J. (1987). Cognitive vulnerability to depression: An investigation of two hypotheses. The British Journal of Clinical Psychology, 26, 113-126.

Watkins, E., \& Moulds, M. (2005). Distinct modes of ruminative selffocus: Impact of abstract versus concrete rumination on problem solving in depression. Emotion (Washington, DC), 5, 319-328. doi:10.1037/1528-3542.5.3.319.

Watkins, E., \& Teasdale, J. D. (2001). Rumination and overgeneral memory in depression: Effects of self-focus and analytic thinking. Journal of Abnormal Psychology, 110, 353-357. doi: 10.1037/0021-843X.110.2.333.

Watkins, E., \& Teasdale, J. D. (2004). Adaptive and maladaptive selffocus in depression. Journal of Affective Disorders, 82, 1-8. doi: 10.1016/j.jad.2003.10.006. 